\title{
EFECTO DEL COLOR Y DE LA DENSIDAD DEL POLIETILENO DE FUNDAS PARA CUBRIR EL RACIMO SOBRE DIMENSIONES, PRESENTACIÓN Y CALIDAD POSCOSECHA DE FRUTOS DE BANANO Y PLÁTANO
}

\author{
Alfonso Vargas ${ }^{1 / *}$, Henry Valle ${ }^{*}$, Miguel González* \\ Palabras clave: Musa AAA, Musa AAB, protección del racimo, subgrupo Cavendish, subgrupo Falso Cuerno. \\ Keywords: Musa AAA, Musa AAB, Bunch protection, Cavendish subgroup, False Horn subgroup.

\begin{abstract}
RESUMEN
Se evaluó el efecto del color (azul, verde y rojo) y densidad (baja y alta) del polietileno de fundas protectoras del racimo de Musa en 4 experimentos de banano (Musa AAA) y en 1 de plátano (Musa AAB) realizados en fincas comerciales del Caribe de Costa Rica. El peso del racimo y el intervalo de días del embolse a la cosecha no difirieron entre los colores $(p>0,4116)$ ni entre las densidades ( $p>0,2583$ ) de las fundas. La apariencia del racimo presentó diferencias entre colores $(p<0,0067)$ y entre la densidades del polietileno $(\mathrm{p}<0,0211)$ solamente en el experimento de plátano, donde el azul y el rojo no variaron en promedio, pero sí ambos del verde. En este experimento, el mayor porcentaje de racimos sin manchas o lesiones se obtuvo con la bolsa de alta densidad. El grosor del fruto central de la fila externa de la segunda, cuarta y sexta mano fue similar entre los colores ( $p>0,0669)$ y densidades ( $p>0,2370)$ de las fundas. La longitud del fruto central de las manos antes mencionadas tampoco presentó diferencias entre colores ( $p>0,1446)$ y solo varió con la densidad en la segunda y cuarta mano del experimento 3 $(\mathrm{p}<0,0501)$, cuya longitud fue mayor con la funda
\end{abstract}

1 Autor para correspondencia. Correo electrónico alfvarga@corbana.co.cr

\begin{abstract}
Effect of color and density of polyethylene bunch covers on the dimensions, appearance and postharvest quality of banana and plantain fruits. The effect of the color (blue, green and red) and of the density (low and high) of polyethylene bunch covers was evaluated in 4 experiments on banana (Musa AAA) and 1 on plantain (Musa AAB) at commercial farms in the Caribbean region of Costa Rica. The bunch weight and the interval of days from bagging to harvest did not differ between colors $(p>0.4116)$ nor between densities $(p>0.2583)$ of the bunch covers. The bunch appearance showed differences among colors $(\mathrm{p}<0.0067)$ and among polyethylene densities $(\mathrm{p}<0.0211)$ only in the plantain experiment, where blue and red did not vary on the average, but both did from the green one. In this experiment, the greater percentage of bunches without spots or injuries was obtained with the high density cover. The thickness of the central fruit of the outer row in the second, fourth and sixth hand was similar between colors ( $p>0.0669)$ and densities ( $p>0.2370)$ of the covers. The length of the central fruit of those hands did not present differences between colors
\end{abstract}

* Corporación Bananera Nacional. Costa Rica. 
de alta densidad. El color y la firmeza de la cáscara, la firmeza del fruto y el porcentaje de sólidos totales (brix) fueron similares entre los colores ( $>00,0899)$ y entre las densidades ( $p>0,0606)$ de las fundas. El porcentaje de acidez presentó diferencias $(p<0,0001)$ entre colores solamente en el experimento 1. Los resultados indican que generalmente, bajo las condiciones agroclimáticas y de manejo propias de Caribe costarricense, no hubo efecto del color ni de la densidad del polietileno evaluados sobre los racimos y frutos de banano y plátano.

\section{INTRODUCCIÓN}

La práctica del embolse al racimo de la planta de banano se inició en la zona atlántica de Costa Rica en forma experimental en 1963 con el propósito de controlar mecánicamente las infestaciones del trips de la mancha roja (Chaetanaphothrips orchidii y C. signipennis). Las primeras pruebas durante ese año consistieron en determinar el mejor diseño de la bolsa en relación con sus dimensiones, grosor del polietileno y diámetro de las perforaciones. Estas primeras observaciones mostraron que la práctica era sumamente prometedora (Lara 1970). Actualmente, el uso de fundas cobertoras de polietileno al racimo de banano es una práctica común en la actividad bananera y la labor es considerada esencial para mejorar el rendimiento y la presentación en las plantaciones comerciales dedicadas a la exportación (Daniells et al. 1992, Soto 1992, Johns 1996, Robinson 1996).

La funda de polietileno protege al racimo contra bajas temperaturas, plagas y del efecto abrasivo de hojas y productos químicos (Daniells et al. 1992, Robinson 1996, Chillet y Jannoyer 1996). La reducción del intervalo
( $>0.1446)$ and only varied with cover density in the second and fourth hand of experiment 3 $(\mathrm{p}<0.0501)$, whose length value was greater with the high density cover. The fruit peel color and firmness, the fruit firmness and percentage of total solids (brix) were similar between the colors $(p>0.0899)$ and between the densities $(p>0.0606)$ of the covers. The acidity percentage showed differences $(p<0.0001)$ between colors only in experiment 1 . The results indicate that under the agro-climatic and management conditions of the Caribbean region of Costa Rican, there was in general no effect of the color nor of the polyethylene density evaluated on banana and plantain bunches and fruits.

floración-cosecha, el aumento del largo y diámetro de los frutos y del peso del racimo, con respecto a los racimos sin enfundar (Campbell y Williams 1976, Daniells et al. 1987, Lara 1970), pese a que fueron resultados secundarios, crearon mayor expectación e hicieron que el embolse se universalizara en el mundo bananero (Soto 1992). La reducción de días a cosecha también fue determinada por Daniells et al. 1992 pero, el peso de racimo solo se incrementó (9\%), cuando la parte terminal de la funda se anudó. Daza y Cayón (2004) indican que la selección apropiada del color del polietileno empleado para embolsar los racimos de banano y plátano puede ejercer una influencia determinante sobre el desarrollo y la presentación comercial de los frutos de Musa.

El color del polietileno actúa como filtro de la RFA y al respecto, Cayón (2007) indica que bajo una condición de RFA incidente de 2298 $\mu \mathrm{mol} \mathrm{s}{ }^{-1} \mathrm{~m}^{-2}$ menciona que el polietileno de color rojo o amarillo permite la transmisión al interior de las fundas de 28 y $25 \%$, de la RFA respectivamente. El polietileno de color negro solo transmite un minúsculo porcentaje de la RFA $(0,1 \%)$, el de color azul permite la transmisión del $73 \%$ de las longitudes de onda de la RFA hacia 
el interior de la funda y el polietileno sin color (transparente) transmite el 93,5 de la RFA incidente. La transmisión e interceptación de la RFA en el polietileno de color blanco fue intermedio al del color azul o aquel sin color mientras que el polietileno de color verde transmite el $13,4 \%$ de la RFA.

Robinson (2006) en banano evaluó diferentes colores de fundas y solo encontró una respuesta productiva al color azul, dado que según dicho autor, este color permite la transmisión de calor pero reduce el daño por la quemadura de sol. Asimismo, Galán Sauco et al (1996) con bananos del subgrupo Cavendish (Musa AAA, cv. Pequeña Enana) y bajo condiciones subtropicales de las Islas Canarias indican también que las fundas de color azul se comportaron mejor que los otros colores evaluados (blanco y blanco-plateado). Sin embargo, Chillet y Jannoyer (1996) y Jannoyer y Chillet (1998) en las Antillas Francesas determinaron bananos del subgrupo Cavendish (Grande Naine) que los racimos embolsados con fundas de color azul no fueron superiores productivamente con aquellos provenientes de fundas sin color con aditivos reductores de luz unltravioleta e infrarroja. De igual manera Cayón (2006) en bananos del subgrupo Cavendish encontró que las fundas de color azul no tuvieron un efecto sobre el peso del racimo. El anterior autor añade que el peso del racimo y el peso del fruto fue mayor con fundas de color verde (alta densidad del polietileno) mientras que las fundas de color rojo lo fueron solo para la segunda variable mencionada. En plátano Cayón (2007) determinó que los racimos de mayor peso fueron obtenidos con los colores verde, azul o blanco y que el primero de ellos (verde), superó en un $15 \%$ el valor que presentaron para esta variable las fundas de color rojo, negro o amarillo y sin color. Al respecto, tanto en banano como en plátano Daza y Cayón (2004) mencionan que tanto para banano como plátano el peso del racimo, la longitud y grosor del fruto de la segunda y última mano y el peso fresco del fruto de la segunda mano fueron mayores con el uso de fundas de color verde, rojo o azul.
Soto (1992) evaluó en banano fundas de polietileno de color azul, verde, rosado y sin color. De acuerdo con el citado autor, el color azul, aunque disminuyó la intensidad del color verde del fruto, no presentó efecto importante en su presentación; los colores verde y rosado produjeron frutos de color pálido con cutículas muy sensibles a golpes y la funda sin color mantuvo el fruto más verde y con una cáscara más resistente. Chillet y Jannoyer (1996) con fundas sin color (transparentes) y de color azul no determinaron en bananos del subgrupo Cavendish (Grande Naine) ubicados en plantaciones comerciales de las Antillas un efecto negativo sobre la firmeza de la cáscara, en contraste con la disminución que encontró en dicha variable Heenan (1973) con el enfundado de racimos de banano de cultivares (Grande Naine y Dwarf Cavendish) del mismo subgrupo (Cavendish).

Daza y Cayón (2006) en banano encontraron con las fundas de color amarillo o rojo una mayor concentración de almidón en la pulpa, y, de azúcares, con las fundas sin color (transparentes) o de color azul. En plátano de acuerdo con Cayón (2007) los frutos que se desarrollaron a partir de fundas de color azul, transparente o blanco presentan concentraciones superiores de azúcares totales al momento de la cosecha. Al respecto, Daza y Cayón (2004) mencionan que las fundas de color azul (baja y alta densidad del polietileno) en banano, y las fundas de color amarillo o azul (baja densidad del polietileno) en plátano, aceleraron la madurez fisiológica del fruto.

Adicionalmente, Daza y Cayón (2004) encontraron que las fundas de color verde o rojo (alta densidad de polietileno) en banano y las de color verde o azul (alta densidad del polietileno) en plátano contribuyeron a mantener un equilibrio en las concentraciones de azúcares durante el desarrollo del fruto.

En la actualidad, la funda de color azul es universalmente usada en la actividad bananera y platanera. No obstante, fundas de otros colores principalmente de color rojo o verde, comienzan a utilizarse en plantaciones comerciales de ambos 
cultivos, de acuerdo con nuevas expectativas de producción.

El objetivo del presente trabajo fue el de comparar, bajo condiciones tropicales del Caribe de Costa Rica, el efecto del color (azul, rojo y verde) y de la densidad (baja y alta) del polietileno usado en fundas protectoras del racimo sobre las dimensiones, la presentación y la calidad poscosecha de los frutos de banano (Musa AAA, cvs. Grande Naine y Williams) y de plátano (Musa AAB, cv. Hartón Enano).

\section{MATERIALES Y MÉTODOS}

Durante el año 2008 y enero del 2009 se realizaron en el Caribe de Costa Rica, provincia de Limón, 4 experimentos en banano (Musa AAA) en los cantones de Matina (finca El Esfuerzo, experimentos 1 y 4), Siquirres (finca Verde Azul, experimento 2) y Pococí (finca La Teresa, experimento 3) y 1 experimento en plátano (Musa AAB) localizado en la provincia de Heredia, cantón de Sarapiquí (Platanera Don Rigo, experimento 5). Los experimentos 1, 2 y 4 se ubicaron en suelos de origen sedimentario ubicados al este del río Reventazón (Zona Este) y los experimentos 3 y 5 se localizaron en suelos de origen volcánico ubicados al oeste del río Reventazón (Zona Oeste). En banano, con excepción del experimento 4 (cv. Williams) los restantes experimentos fueron realizados en áreas sembradas con el cv. Grande Naine. En plátano el experimento se efectuó en un área sembrada con el cultivar Hartón Enano.

En las fincas ubicadas en la zona Este (experimentos 1, 2 y 4) la precipitación total durante el período experimental fue de 397 , 592 y $1320 \mathrm{~mm}$, las temperaturas máxima media y mínima media fueron de 29,7 y 21,4 ; 29,5 y 22,3 y 27,5 y $21,4{ }^{0} \mathrm{C}$ y la humedad relativa promedio fue de 90,$1 ; 92,1$ y 94,0 ; respectivamente. En las fincas situadas en la zona Oeste (experimentos 3 y 5) ambos trabajos se realizaron de manera simultánea. La precipitación total del período experimental fue de $1294 \mathrm{~mm}$, las temperaturas promedio máxima y mínima fueron de 32,4 y $22,2{ }^{0} \mathrm{C}$ y la humedad relativa promedio fue de $86,1 \%$.

Las fincas en donde se desarrollaron los experimentos 1, 2 y 4 (banano) fueron sembradas a inicio de los años 90 con el cv. Grande Naine. La finca del experimento 3 (banano) fue sembrada en 1963 con el cv. Valery y el área en donde se realizó el estudio fue renovada en el año 1998 con el cv. Williams. Las fincas de banano presentan una densidad de población promedio de 1750 plantas.ha $^{-1}$. La finca en donde se efectuó el experimento 5 (plátano) se sembró en el 2008 con una densidad de población de 2222 plantas.ha $^{-1}$.

La fertilización en los experimentos de banano consideró principalmente la adición ( $\mathrm{kg}$. $\mathrm{ha}^{-1}$ ), de acuerdo a la zona (Este u Oeste) de entre 375 a 400 de N, 50 a 60 de $\mathrm{P}_{2} \mathrm{O}_{5}, 400-500 \mathrm{~kg}$ de $\mathrm{K}_{2} \mathrm{O}, 100$ a 150 de $\mathrm{CaO}$ y 75-100 de $\mathrm{MgO}$ fraccionados en 12 a 17 ciclos. En el experimento de plátano la fertilización se efectuó con $70 \mathrm{~g}$ de $\mathrm{N}$ y 84 g.planta $^{-1}$ de $\mathrm{K}_{2} \mathrm{O}$ fraccionado en 5 ciclos.

El combate de la Sigatoka negra (Mycosphaerella fijiensis) en las fincas de banano se realizó mediante la aspersión aérea de fungicidas sistémicos y protectores. Los mismos fueron aplicados rotativamente y en mezcla con aceite mineral (5-10 1.ha- $\left.{ }^{-1}\right)$ de acuerdo con los requerimientos y estrategias de combate propias de cada finca y época climática para un promedio anual de 48 ciclos de aplicación. En la finca de plátano se efectuó con bomba manual mediante la aspersión de fungicidas protectores en mezcla con aceite mineral $\left(41 . \mathrm{ha}^{-1}\right)$ con un total de 10 ciclos de aplicación por ciclo de cultivo. Operaciones semanales de deshoja sanitaria se utilizaron como complemento al combate químico.

En cada finca se estudió un grupo de inflorescencias que emergieron en una misma semana y fueron embolsadas 2 semanas después, en conjunto con la eliminación de la mano falsa y generalmente de la chira. No se realizó el desdede de frutos laterales en ninguno de los casos y la desflora en el campo solo se practicó en las fincas de los experimentos 2, 3 y 4 . Al embolse, entre las manos 2 y 3 , se anudó al raquis una cinta de polipropileno impregnada con clorpirifos al $1 \%$ 
y 6 semanas después se repitió la operación en la sección inferior del raquis correspondiente a la última mano cubierta por la funda.

Los tratamientos definidos por el color y la densidad del polietileno fueron los siguientes: 1-azul-baja densidad, 2-azul-alta densidad, 3-rojo-baja densidad, 4 rojo-alta densidad, 5 -verde baja densidad y 6-verde alta densidad. Las características de las fundas y del polietileno se presentan en el Cuadro 1. Los tratamientos se aplicaron, de manera aleatoria, en grupos de racimos de similar edad y número de manos a la floración, de acuerdo con la aparición de los tamaños de racimo requerido. En este estudio no se consideró la inclusión de un tratamiento sin embolsar toda vez que Robinson (1992), Soto (1992) y Lara (1970) coinciden en que tanto la productividad como la calidad de los racimos se incrementa significativamente con el uso de fundas de polietileno con respecto a aquellos sin embolsar. Bajo esta perspectiva y dado que en todas las operaciones mundiales destinadas a producir frutos para la exportación el embolse de los racimos es una práctica establecida, se estimó que la inclusión de un tratamiento sin embolse del racimo, cuyas desventajas son muchas y conocidas, no sería procedente.

Los tamaños de racimo a partir de manos verdaderas a la floración y el desmane de acuerdo con el experimento 1(uno) fueron: 9 manos y remoción de 3 de ellas, 10 manos y remoción de 3 de ellas para los experimentos 2 y 4, 9 y 10 manos y remoción de 2 y 3 de ellas, respectivamente para el experimento 3 y sin desmane para el experimento 5. Cada planta y su racimo se considero como una repetición. El número de racimos por tratamiento varió de 21 a 23 (exp.1), de 29 a 31 (exp. 2), de 14 a 16 (exp. 3), de 19 a 20 (exp. 4) y de 21 a 24 (exp. 5).

La cosecha se realizó en los experimentos de banano a partir de una edad preestablecida (10-11 semanas del embolse) y en etapas o semanas sucesivas (2 a 3) mediante la determinación de un grosor preestablecido del fruto central de la fila externa de la segunda mano de 44 a 45 treintaidosavos de pulgada en los experimentos 1,2 y $3 \mathrm{y}$, de 37 treintaidosavos de pulgada en el fruto central de la fila externa de la última mano, en el experimento 4. En el experimento 5 (plátano), la cosecha se inició a las 10 semanas del embolse, durante 3 etapas o semanas sucesivas y con un grosor preestablecido del fruto central de la fila externa de la segunda mano de 52 treintaidosavos de pulgada en el fruto central de la fila externa de la segunda mano.

Los racimos de los experimentos 1, 2, 3 y 5 se cosecharon en la época climática favorable y el experimento 4 en la época climática adversa para el crecimiento de la planta y el desarrollo del racimo, ambas mencionadas por Vargas y Blanco (2004) Serrano et al (2008). La época favorable estuvo constituida por los períodos del año

Cuadro 1. Características físicas de las fundas de polietileno usadas en la protección de racimos de banano (Musa AAA subgrupo Cavendish) y plátano (Musa AAB, subgrupo Falso Cuerno).

\begin{tabular}{|c|c|c|c|c|c|}
\hline Color & Densidad & $\begin{array}{l}\text { Grosor } \\
(\mu)^{1}\end{array}$ & Perforación (mm) & $\begin{array}{l}\text { Ancho } \\
(\mathrm{cm})\end{array}$ & $\begin{array}{c}\text { Insecticida de impregnación } \\
(\%)\end{array}$ \\
\hline Azul & Baja & 12,7 & 4,0 & 81,3 & bifentrina 0,1 \\
\hline Azul & Alta & 12,7 & 4,0 & 81,3 & bifentrina 0,1 \\
\hline Rojo & Baja & 10,2 & 6,0 & 81,3 & clorpirifos 1,0 \\
\hline Rojo & Alta & 12,7 & 4,0 & 81,3 & bifentrina 0,1 \\
\hline Verde & Baja & 12,7 & 6,0 & 81,3 & clorpirifos 1,0 \\
\hline Verde & Alta & 12,7 & 6,0 & 81,3 & clorpirifos 1,0 \\
\hline
\end{tabular}

${ }^{1 /}=0,001 \mathrm{~mm}$. Una milésima de pulgada $=0,0254 \mathrm{~mm}$. 
lluvioso y frío (noviembre, diciembre y enero) y moderadamente seco y caliente (febrero, marzo y abril). La época adversa por su parte estuvo determinada por los períodos del año caliente y lluvioso (mayo, junio y julio) y seco y fresco (agosto, setiembre y octubre).

Las variables medidas fueron: radiación fotosintéticamente activa (RFA) tanto incidente como transmitida $\left(\mu \mathrm{mol} \mathrm{s}{ }^{-1} \mathrm{~m}^{-2}\right)$, peso del racimo $(\mathrm{kg})$, número de días desde el embolse a la cosecha, apariencia del racimo, grosor (treintaidosavos de pulgada en banano, mm en plátano) y longitud $(\mathrm{cm})$ del fruto central de la fila externa de la segunda, cuarta y sexta mano, firmeza de la cáscara del fruto lateral de la fila externa de la segunda mano (en grado 1 de maduración) y el color de la cáscara del fruto central de la segunda mano (grado 1 de maduración) según escala $L^{*}, a^{*}, b^{*}$.

La RFA incidente se midió sobre el dosel de la planta y la RFA transmitida se determinó dentro de la funda solamente en los experimentos 1 y 3. Los datos de la RFA se almacenaron en un dataLogger modelo LI-1000 de LI-COR ${ }^{\circledR}$, al cual se le adicionó un censor Quantum LI-190SA (LI$\mathrm{COR}^{\circledR}$ ) el cual determina la densidad del flujo de fotones $\left(\mu \mathrm{mol} \mathrm{s}{ }^{-1} \mathrm{~m}^{-2}\right)$. El número de repeticiones fue de 32 y 24, en los experimentos 1 y 3, para cada una de las RFA medidas.

El peso del racimo y del pinzote se registró con la ayuda de una romana con capacidad para $50 \mathrm{~kg}$ graduada cada $200 \mathrm{~g}$. El grosor del fruto se midió en la parte media, perpendicularmente al plano de la curvatura, con la ayuda de un calibrador graduado en treintaidosavos de pulgada ( 1 unidad o grado $=0,794 \mathrm{~mm}$ ) en el caso de banano y con un vernier graduado en $\mathrm{mm}$ en el caso de plátano. La longitud se midió a lo largo de la parte externa, desde la zona de unión del pedúnculo con la pulpa, hasta el ápice (de "pulpa a punta") con una cinta métrica de plástico graduada en mm. La medición del color de la cáscara se efectuó mediante un colorímetro portátil AccuProbe ${ }^{\circledR}$ que determinó los valores de $L^{*}, a^{*}$, $b^{*}$ de acuerdo con la escala de Hunter en donde la coordenada " $L$ " es una medida de la claridad (blanco-negro y varía desde el punto de ausencia de reflejo, $\mathrm{L}=0$, hasta el punto de reflejo difuso perfecto, $L=100$ ). La escala "a" varía desde los valores negativos para el color verde, hasta los positivos para el rojo. La escala "b" varía desde los valores negativos para el color azul, hasta los positivos para el amarillo. La apariencia del racimo se registró mediante una escala que consideró las siguientes condiciones: 1-sin manchas o lesiones, 2 con pocas manchas o lesiones pequeñas, 3-con pocas manchas o lesiones grandes, 4-con muchas manchas o lesiones pequeñas, 5-con muchas manchas o lesiones grandes, 6-totalmente cubierto de manchas o lesiones (tejido necrosado).

La medición de la firmeza de la cáscara en grado 1 de maduración (escala de Von Loesecke (1950)) se efectuó en un fruto lateral izquierdo de la segunda mano de racimos de banano tomados al azar con la ayuda de un penetrómetro Chatillon ${ }^{\circledR}$ de punta cónica (Newtons) en los experimentos 1,2 y 3 y con un penetrómetro Wagner ${ }^{\circledR}$ con punta cónica (libras) en los experimentos 4 y 5. El número de repeticiones varió de 6 a 10 .

Para la determinación de las variables poscosecha medidas en grado 5 de maduración (escala de Von Loesecke (1950): firmeza del fruto, grados brix (\%) y acidez titulable expresada como \% de ácido málico), medidas en grado 5 de maduración para los experimentos de banano (1 a 4) se procesaron 2 gajos centrales de la segunda mano en racimos tomados al azar. Posteriormente fueron empacados en cajas de cartón corrugado y transportados a una cámara de frío para su almacenamiento (7 días- $\left.14^{\circ} \mathrm{C}\right)$, y posterior aplicación de etileno y maduración $\left(18^{\circ} \mathrm{C}\right)$. La firmeza del fruto se determinó mediante la compresión de la cascara y pulpa de éste, tanto en la parte apical como distal, con un penetrómetro Effegi ${ }^{\circledR}$ con punta plana (libras).

Los valores de firmeza obtenidos en libras fueron convertidos a Newtons mediante su multiplicación por un factor de 4,4448. Los grados Brix se determinaron mediante un refractómetro digital (Atago Palette 100) y la acidez con un un titulador automático (Orion 960); en concordancia 
con la metodología descrita por Dadzie y Orchard (1997). El número de repeticiones varió de 6 a 8 .

El conjunto de datos correspondiente a RFA transmitida se analizó bajo un modelo factorial de 2 fincas x 2 lugares de medición $x$ 3 colores (azul, rojo y verde) x 2 densidades de polietileno (baja y alta). Los datos de la RFA incidente se expresaron como un promedio de referencia.

Los datos de crecimiento y producción de los experimentos 1 a 4 (banano) se analizaron con un modelo factorial de 4 fincas x 3 colores x 2 densidades de polietileno y los correspondientes al experimento 5 (plátano), por provenir de una sola finca, como un factorial de 3 colores x 2 densidades del polietileno. Los datos correspondientes a los diferentes grados de apariencia del racimo fueron analizados mediante la Prueba de chi-cuadrado de Cochran-Mantel-Haenszl.

\section{RESULTADOS}

Independientemente del conjunto de fincas y de la densidad del polietileno (interacción finca $\mathrm{x}$ densidad: $\mathrm{p}=0,2564)$, las fundas de color azul (RFA transmitida $=284 \mu \mathrm{mol} \mathrm{s} \mathrm{s}^{-1} \mathrm{~m}^{-2}$ ) permitieron un mayor paso de luz al racimo $(\mathrm{p}=0,0001)$ que las de color rojo (RFA transmitida $=240 \mu \mathrm{mol} \mathrm{s}^{-1} \mathrm{~m}^{-}$ 2) y verde (RFA transmitida $=238 \mu \mathrm{mol} \mathrm{s}^{-1} \mathrm{~m}^{-2}$ ). Independientemente del color y del conjunto de fincas (interacción finca $x$ color: $p=0,9749$ ), las fundas de baja densidad de polietileno (RFA transmitida $\left.=285 \mu \mathrm{mol} \mathrm{s}^{-1} \mathrm{~m}^{-2}\right)$ permitieron un mayor paso de luz al racimo $(\mathrm{p}=0,0001)$ que aquellas de alta densidad (RFA transmitida $\left.=223 \mu \mathrm{mol} \mathrm{s}^{-1} \mathrm{~m}^{-2}\right)$. La RFA incidente fue de $2416 \mu \mathrm{mol} \mathrm{s}^{-1} \mathrm{~m}^{-2}$.

A pesar de que no hubo interacción $(\mathrm{p}<0,0662)$ del color $\mathrm{x}$ densidad del polietileno en la mayoría de las variables correspondientes a las características del racimo, de sus frutos y a la presentación y calidad poscosecha de los mismos, las medias correspondientes se presentan con la consideración de ambos factores, con el propósito de ofrecer al lector una perspectiva más detallada de los resultados.

El peso del racimo y el intervalo de días del embolse a la cosecha (Cuadro 2) no difirieron entre el color $(\mathrm{p}>0,4116)$ ni entre la densidad 8 $\mathrm{p}>0,2583$ ) de las fundas.

La apariencia del racimo (Cuadro 2) solamente presentó diferencias entre colores $(\mathrm{p}<0,0067)$ y entre la densidades del polietileno $(p<0,0211)$ en el experimento 5 (plátano) donde en el azul y el rojo no variaron en promedio, pero sí ambos del verde. En este experimento, el mayor porcentaje de racimos sin manchas o lesiones se obtuvo con la funda de alta densidad.

El grosor del fruto central de la fila externa de la segunda, cuarta y sexta mano (Cuadro 3) fue similar entre los colores $(p>0,0669)$ y densidades $(p>0,2370)$ de las fundas. En la longitud del mismo (Cuadro 4) tampoco hubo diferencias entre colores $(p>0,1446)$ y, entre las densidades de polietileno, solo hubo diferencias en el experimento 3 para el fruto de la segunda $(\mathrm{p}=0,0410)$ y cuarta mano $(p=0,0501)$, valores que fueron mayores con el polietileno de alta densidad.

El color de la cáscara (Cuadro 5) fue similar entre los colores: $L^{*}(p>0,1619)$, $a^{*}$ $(p>0,0968)$ y $b^{*}(p>0,0899)$ y entre densidades: $L^{*}(p>0,0606), a^{*}(p>0,0778)$ y $b^{*}(p>0,1898)$.

La firmeza de la cáscara (Cuadro 6) no difirió entre colores $(p>0,1280)$ ni entre densidades ( $p>0,0964)$. La firmeza del fruto y los grados brix no variaron entre colores $(p>0,1047)$ ni entre densidades $(p>0,0919)$. La acidez titulable presentó diferencias $(\mathrm{p}<0,0001)$ entre colores solo en el experimento 1 , con un mayor valor con el color azul. No hubo diferencias $(p>0,4319)$ entre densidades (Cuadro 6). 
Cuadro 2. Peso, intervalo de embolse a cosecha y apariencia (medias \pm error estándar) de racimos de banano (Musa AAA) y plátano (Musa AAB) embolsados con fundas de polietileno de diferente color y densidad. n=21 a 23 en exp.1, de 29 a 31 en exp. 2, de 14 a 16 en exp. 3, de 19 a 20 en exp. 4 y de 21 a 24 en exp. 5.

\begin{tabular}{|c|c|c|c|c|c|}
\hline \multirow{2}{*}{$\begin{array}{c}\text { Fundas } \\
\left(\text { Color }^{1} \text { y densidad }{ }^{2}\right)\end{array}$} & \multicolumn{5}{|c|}{ Experimentos } \\
\hline & 1 & 2 & 3 & 4 & 5 \\
\hline \multicolumn{6}{|c|}{ Peso del racimo $(\mathrm{kg})$} \\
\hline A y BD & 27,6 & 27,6 & 29,2 & 22,9 & 13,6 \\
\hline A y $\mathrm{AD}$ & 26,6 & 27,8 & 29,2 & 22,9 & 13,0 \\
\hline R y BD & 28,2 & 27,5 & 29,6 & 22,9 & 13,3 \\
\hline R y AD & 27,2 & 27,8 & 30,0 & 22,3 & 13,2 \\
\hline V y BD & 27,0 & 27,9 & 29,4 & 22,5 & 13,8 \\
\hline V y AD & 27,6 & 28,0 & 29,1 & 22,1 & 13,9 \\
\hline Error estándar & 0,5 & 0,4 & 0,7 & 0,6 & 0,5 \\
\hline \multicolumn{6}{|c|}{ ANDEVA (probabilidades) } \\
\hline Color (C ) & 0,4200 & 0,5488 & 0,7421 & 0,5624 & 0,4116 \\
\hline Densidad (D) & 0,6898 & 0,4570 & 0,9713 & 0,5323 & 0,5676 \\
\hline Interacción C x D & 0,0261 & 0,8189 & 0,9207 & 0,8942 & 0,7609 \\
\hline \multicolumn{6}{|c|}{ Intervalo del embolse a la cosecha (días) } \\
\hline A y BD & 73,7 & 74,9 & 86,4 & 81,7 & 73,3 \\
\hline A y $\mathrm{AD}$ & 75,5 & 74,0 & 86,1 & 81,3 & 73,3 \\
\hline R y BD & 74,3 & 75,1 & 85,3 & 81,7 & 73,0 \\
\hline R y AD & 73,3 & 75,4 & 85,7 & 81,5 & 72,2 \\
\hline V y BD & 73,6 & 74,7 & 85,4 & 82,4 & 72,8 \\
\hline V y AD & 73,6 & 77,0 & 86,4 & 82,5 & 74,0 \\
\hline Error estándar & 0,8 & 0,7 & 1,0 & 0,8 & 1,1 \\
\hline \multicolumn{6}{|c|}{ ANDEVA (probabilidades) } \\
\hline Color (C) & 0,3237 & 0,2907 & 0,3128 & 0,2800 & 0,9643 \\
\hline Densidad (D) & 0,2583 & 0,3672 & 0,4595 & 0,7036 & 0,6177 \\
\hline Interacción C x D & 0,3396 & 0,1875 & 0,4747 & 0,8915 & 0,8474 \\
\hline \multicolumn{6}{|c|}{ Apariencia del racimo (\% sin manchas o lesiones) } \\
\hline A y BD & 100 & 100 & 100 & 100 & 46 \\
\hline A y AD & 100 & 97 & 100 & 95 & 50 \\
\hline R y BD & 100 & 100 & 100 & 95 & 25 \\
\hline R y AD & 100 & 97 & 93 & 95 & 72 \\
\hline V y BD & 95 & 100 & 100 & 100 & 19 \\
\hline V y AD & 100 & 97 & 100 & 85 & 19 \\
\hline \multicolumn{6}{|c|}{ ANDEVA (probabilidades) } \\
\hline Color (C) & 0,3850 & 0,9989 & 0,4346 & 0,5877 & 0,0067 \\
\hline Densidad (D) & 0,3173 & 0,0833 & 0,3017 & 0,1052 & 0,0211 \\
\hline
\end{tabular}

${ }^{1 /}$ Color del polietileno: $\mathrm{A}=$ azul, $\mathrm{R}=$ rojo, $\mathrm{V}=$ verde. ${ }^{2 / \mathrm{BD}}=$ baja densidad, $\mathrm{AD}=$ alta densidad Notas: En la finca 5 el azul y el rojo no difieren en promedio, pero si ambos con el verde. Solo hubo efecto de la densidad en el color rojo. 
Cuadro 3. Grosor del fruto central de la fila externa de la segunda, cuarta y sexta mano (medias terror estándar) de racimos de banano (Musa AAA) y plátano (Musa AAB) embolsados con fundas de polietileno de diferente color y densidad. n=21 a 23 en exp.1, de 29 a 31 en exp. 2, de 14 a 16 en exp. 3, de 19 a 20 en exp. 4 y de 21 a 24 en exp. 5.

\begin{tabular}{|c|c|c|c|c|c|}
\hline \multirow{2}{*}{$\begin{array}{c}\text { Fundas } \\
\left(\text { Color }^{1} \text { y densidad }\right. \\
\end{array}$} & \multicolumn{5}{|c|}{ Experimentos } \\
\hline & 1 & 2 & 3 & 4 & 5 \\
\hline \multicolumn{6}{|c|}{ Segunda mano (treintaidosavos de pulgada) } \\
\hline A y BD & 45,2 & 45,6 & 46,8 & 42,2 & 52,6 \\
\hline A y $\mathrm{AD}$ & 44,9 & 45,5 & 47,0 & 42,2 & 53,9 \\
\hline R y BD & 45,0 & 45,5 & 47,4 & 42,0 & 52,8 \\
\hline $\mathrm{R}$ y $\mathrm{AD}$ & 45,2 & 45,3 & 47,5 & 41,6 & 53,4 \\
\hline V y BD & 45,3 & 45,4 & 47,4 & 41,8 & 52,4 \\
\hline V y AD & 45,2 & 45,6 & 47,0 & 41,4 & 51,9 \\
\hline Error estándar & 0,2 & 0,2 & 0,3 & 0,3 & 0,4 \\
\hline \multicolumn{6}{|c|}{ ANDEVA (probabilidades) } \\
\hline Color (C) & 0,7631 & 0,5238 & 0,3776 & 0,2890 & 0,0669 \\
\hline Densidad (D) & 0,7785 & 0,6979 & 0,9658 & 0,2831 & 0,2370 \\
\hline Interacción C x D & 0,5746 & 0,5770 & 0,7270 & 0,8740 & 0,2228 \\
\hline \multicolumn{6}{|c|}{ Cuarta mano (treintaidosavos de pulgada) } \\
\hline A y BD & 44,0 & 44,0 & 45,2 & 40,7 & 52,7 \\
\hline A y AD & 43,7 & 43,8 & 45,8 & 40,9 & 51,4 \\
\hline R y BD & 43,8 & 44,0 & 45,4 & 40,8 & 52,6 \\
\hline R y AD & 44,2 & 43,8 & 45,9 & 40,2 & 52,2 \\
\hline V y BD & 44,0 & 44,1 & 45,4 & 40,4 & 51,3 \\
\hline V y AD & 43,9 & 44,2 & 45,4 & 40,5 & 52,3 \\
\hline Error estándar & 0,3 & 0,2 & 0,3 & 0,3 & 0,4 \\
\hline \multicolumn{6}{|c|}{ ANDEVA (probabilidades) } \\
\hline Color (C) & 0,6832 & 0,4980 & 0,6853 & 0,5394 & 0,5113 \\
\hline Densidad (D) & 0,9745 & 0,4832 & 0,6449 & 0,7007 & 0,6082 \\
\hline Interacción C x D & 0,3173 & 0,8500 & 0,8443 & 0,4605 & 0,1011 \\
\hline \multicolumn{6}{|c|}{ Sexta mano (treintaidosavos de pulgada) } \\
\hline A y BD & 42,3 & 42,8 & 44,3 & 40,0 & 52,0 \\
\hline A y $A D$ & 42,3 & 42,6 & 43,7 & 39,7 & 51,8 \\
\hline R y BD & 42,3 & 42,6 & 44,3 & 39,8 & 52,0 \\
\hline R y AD & 42,5 & 42,8 & 44,2 & 39,2 & 51,7 \\
\hline V y BD & 42,3 & 42,6 & 44,3 & 39,1 & 51,0 \\
\hline V y AD & 42,3 & 42,7 & 44,0 & 39,4 & 51,8 \\
\hline Error estándar & 0,3 & 0,2 & 0,3 & 0,3 & 0,6 \\
\hline \multicolumn{6}{|c|}{ ANDEVA (probabilidades) } \\
\hline Color (C ) & 0,6908 & 0,9961 & 0,8106 & 0,2121 & 0,7361 \\
\hline Densidad (D) & 0,7541 & 0,9435 & 0,3346 & 0,3339 & 0,8644 \\
\hline Interacción C x D & 0,8468 & 0,7738 & 0,7977 & 0,7415 & 0,7415 \\
\hline
\end{tabular}

1/Color del polietileno: $\mathrm{A}=$ azul, $\mathrm{R}=$ rojo, $\mathrm{V}=$ verde. ${ }^{2 / \mathrm{BD}}=$ baja densidad, $\mathrm{AD}=$ alta densidad. 
Cuadro 4. Longitud (pulpa a punta) del fruto central de la fila externa de la segunda, cuarta y sexta mano (medias \pm error estándar) de racimos de banano (Musa AAA) y plátano (musa AAB) embolsados con fundas de polietileno de diferente color y densidad. n=21 a 23 en exp.1, de 29 a 31 en exp. 2, de 14 a 16 en exp. 3, de 19 a 20 en exp. 4 y de 21 a 24 en exp. 5.

\begin{tabular}{|c|c|c|c|c|c|}
\hline \multirow{2}{*}{$\begin{array}{c}\text { Fundas } \\
\left(\text { Color }^{1} \text { y densidad }\right.\end{array}$} & \multicolumn{5}{|c|}{ Experimentos } \\
\hline & 1 & 2 & 3 & 4 & 5 \\
\hline \multicolumn{6}{|c|}{ Segunda mano $(\mathrm{cm})$} \\
\hline A y BD & 25,2 & 25,9 & 24,4 & 24,0 & 25,3 \\
\hline A y $A D$ & 25,0 & 25,9 & 24,7 & 24,3 & 25,0 \\
\hline R y BD & 24,7 & 25,7 & 24,2 & 24,0 & 25,5 \\
\hline R y AD & 25,3 & 25,6 & 24,8 & 24,1 & 24,4 \\
\hline V y BD & 25,0 & 25,7 & 24,3 & 24,3 & 24,7 \\
\hline $\mathrm{V}$ y $\mathrm{AD}$ & 25,2 & 26,1 & 24,7 & 23,7 & 24,2 \\
\hline Error estándar & 0,2 & 0,2 & 0,3 & 0,3 & 0,4 \\
\hline \multicolumn{6}{|c|}{ ANDEVA (probabilidades) } \\
\hline Color (C) & 0,8905 & 0,5348 & 0,9039 & 0,8810 & 0,2456 \\
\hline Densidad (D) & 0,2742 & 0,6352 & 0,0501 & 0,7827 & 0,0696 \\
\hline Interacción C x D & 0,1639 & 0,4509 & 0,7354 & 0,1789 & 0,6650 \\
\hline \multicolumn{6}{|c|}{ Cuarta mano $(\mathrm{cm})$} \\
\hline A y BD & 23,1 & 24,8 & 23,3 & 22,7 & 24,6 \\
\hline A y AD & 23,1 & 24,2 & 23,7 & 23,2 & 24,1 \\
\hline R y BD & 23,5 & 24,5 & 23,3 & 22,9 & 24,0 \\
\hline R y AD & 23,7 & 24,4 & 23,4 & 22,9 & 23,6 \\
\hline $\mathrm{V}$ y BD & 23,1 & 24,4 & 23,0 & 23,1 & 24,0 \\
\hline $\mathrm{V}$ y AD & 23,2 & 25,1 & 23,6 & 22,6 & 23,7 \\
\hline Error estándar & 0,2 & 0,2 & 0,3 & 0,3 & 0,4 \\
\hline \multicolumn{6}{|c|}{ ANDEVA (probabilidades) } \\
\hline Color $(\mathrm{C})$ & 0,0947 & 0,4077 & 0,4544 & 0,9129 & 0,4124 \\
\hline Densidad (D) & 0,6945 & 0,9017 & 0,0410 & 0,9080 & 0,2126 \\
\hline Interacción C x D & 0,9116 & 0,0296 & 0,9146 & 0,1623 & 0,9618 \\
\hline \multicolumn{6}{|c|}{ Sexta mano $(\mathrm{cm})$} \\
\hline A y BD & 21,7 & 22,9 & 21,6 & 21,7 & 22,7 \\
\hline A y $A D$ & 21,7 & 22,8 & 21,4 & 21,4 & 22,0 \\
\hline $\mathrm{R}$ y $\mathrm{BD}$ & 21,7 & 22,7 & 21,1 & 21,5 & 22,5 \\
\hline R y AD & 21,9 & 22,7 & 21,8 & 21,3 & 22,4 \\
\hline $\mathrm{V}$ y BD & 21,5 & 23,0 & 21,7 & 21,6 & 22,0 \\
\hline $\mathrm{V}$ y $\mathrm{AD}$ & 21,4 & 22,9 & 22,2 & 21,4 & 22,4 \\
\hline Error estándar & 0,2 & 0,2 & 0,3 & 0,2 & 0,4 \\
\hline \multicolumn{6}{|c|}{ ANDEVA (probabilidades) } \\
\hline Color $(\mathrm{C})$ & 0,3047 & 0,4083 & 0,1446 & 0,8598 & 0,7596 \\
\hline Densidad (D) & 0,6853 & 0,8686 & 0,1283 & 0,2068 & 0,8040 \\
\hline Interacción C x D & 0,5555 & 0,9287 & 0,2309 & 0,9810 & 0,2612 \\
\hline
\end{tabular}

1/Color del polietileno: $\mathrm{A}=$ azul, $\mathrm{R}=$ rojo, $\mathrm{V}=$ verde. ${ }^{2 /} \mathrm{BD}=$ baja densidad, $\mathrm{AD}=$ alta densidad 
Cuadro 5. L*a*b* del fruto central de la fila externa de la segunda mano (medias \pm error estándar) de racimos de banano (Musa AAA) y plátano (Musa AAB) embolsados con fundas de polietileno de diferente color y densidad. n=21 a 23 en exp.1, de 29 a 31 en exp. 2, de 14 a 16 en exp. 3, de 19 a 20 en exp. 4 y de 21 a 24 en exp. 5.

\begin{tabular}{|c|c|c|c|c|c|}
\hline \multirow{2}{*}{$\begin{array}{c}\text { Fundas } \\
\left(\text { Color }^{1} \text { y densidad }\right. \\
\end{array}$} & \multicolumn{5}{|c|}{ Experimentos } \\
\hline & 1 & 2 & 3 & 4 & 5 \\
\hline \multicolumn{6}{|l|}{ 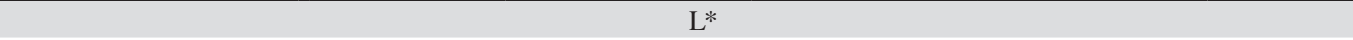 } \\
\hline A y BD & 54,59 & 54,39 & 54,29 & 55,38 & 49,36 \\
\hline A y $\mathrm{AD}$ & 53,38 & 54,45 & 53,62 & 53,26 & 48,20 \\
\hline R y BD & 53,08 & 54,41 & 53,19 & 54,10 & 49,10 \\
\hline R y AD & 54,39 & 54,73 & 54,90 & 55,90 & 48,93 \\
\hline V y BD & 54,15 & 53,50 & 53,67 & 54,21 & 50,48 \\
\hline V y AD & 55,06 & 54,70 & 53,16 & 55,28 & 48,42 \\
\hline Error estándar & 0,48 & 0,41 & 0,60 & 0,52 & 0,69 \\
\hline \multicolumn{6}{|c|}{ ANDEVA (probabilidades) } \\
\hline Color (C) & 0,1619 & 0,4973 & 0,4027 & 0,5707 & 0,6626 \\
\hline Densidad (D) & 0,3797 & 0,1060 & 0,6611 & 0,6427 & 0,0606 \\
\hline Interacción C x D & 0,0182 & 0,3282 & 0,0283 & 0,0074 & 0,3908 \\
\hline \multicolumn{6}{|c|}{$a^{*}$} \\
\hline A y BD & $-14,55$ & $-14,76$ & $-15,35$ & $-15,47$ & $-12,94$ \\
\hline A y $\mathrm{AD}$ & $-14,94$ & $-14,81$ & $-15,76$ & $-14,79$ & $-13,76$ \\
\hline R y BD & $-14,57$ & $-14,82$ & $-14,77$ & $-15,00$ & $-13,79$ \\
\hline R y AD & $-14,72$ & $-14,91$ & $-15,04$ & $-15,23$ & $-13,97$ \\
\hline V y BD & $-14,47$ & $-14,50$ & $-15,17$ & $-14,84$ & $-14,05$ \\
\hline V y AD & $-15,24$ & $-15,24$ & $-15,51$ & $-15,45$ & $-13,69$ \\
\hline Error estándar & 0,23 & 0,20 & 0,28 & 0,24 & 0,31 \\
\hline \multicolumn{6}{|c|}{ ANDEVA (probabilidades) } \\
\hline Color (C) & 0,6892 & 0,9069 & 0,0968 & 0,9986 & 0,2227 \\
\hline Densidad (D) & 0,9117 & 0,0778 & 0,1814 & 0,7553 & 0,4276 \\
\hline Interacción C x D & 0,5040 & 0,1797 & 0,9737 & 0,0103 & 0,2364 \\
\hline \multicolumn{6}{|c|}{ B } \\
\hline A y BD & 35,12 & 33,40 & 34,44 & 35,23 & 34,25 \\
\hline A y AD & 34,94 & 34,95 & 34,35 & 35,47 & 34,63 \\
\hline R y BD & 33,82 & 33,29 & 34,08 & 34,03 & 36,82 \\
\hline R y AD & 34,79 & 34,62 & 35,38 & 34,95 & 33,78 \\
\hline V y BD & 33,67 & 34,47 & 34,80 & 36,37 & 35,67 \\
\hline V y AD & 32,65 & 33,04 & 35,97 & 35,39 & 35,56 \\
\hline Error estándar & 0,82 & 0,70 & 0,10 & 0,88 & 0,11 \\
\hline \multicolumn{6}{|c|}{ ANDEVA (probabilidades) } \\
\hline Color (C ) & 0,0899 & 0,8395 & 0,4180 & 0,3950 & 0,6008 \\
\hline Densidad (D) & 0,9147 & 0,4007 & 0,1898 & 0,9435 & 0,3250 \\
\hline Interacción C x D & 0,5040 & 0,0662 & 0,5744 & 0,6439 & 0,2448 \\
\hline
\end{tabular}

1/color del polietileno: $\mathrm{A}=$ azul, $\mathrm{R}=$ rojo, $\mathrm{V}=$ verde. ${ }^{2} / \mathrm{BD}=$ baja densidad, $\mathrm{AD}=$ alta densidad. 
Cuadro 6. Firmeza de la cáscara en grado 1 de maduración y firmeza del fruto, grados brix y acidez en grado 5 de maduración (medias \pm error estándar) de frutos de banano (Musa AAA) embolsados con fundas de polietileno de diferente color y densidad, n=6 a 10 en exp.1, 8 en exp. 2, de 6 a 8 en exp. 3 y de 6 a10 en exp. 4.

\begin{tabular}{|c|c|c|c|c|}
\hline \multirow{2}{*}{$\begin{array}{l}\text { Fundas } \\
\left(\text { Color }^{1} \text { y densidad }\right. \\
\end{array}$} & \multicolumn{4}{|c|}{ Experimentos } \\
\hline & 1 & 2 & 3 & 4 \\
\hline \multicolumn{5}{|c|}{ Firmeza de la cascara $(\mathrm{N})$} \\
\hline A y BD & 55,1 & 58,6 & 59,8 & 50,1 \\
\hline A y AD & 57,1 & 54,6 & 60,1 & 49,1 \\
\hline R y BD & 55,3 & 57,0 & 57,1 & 49,0 \\
\hline R y AD & 55,9 & 56,9 & 58,1 & 48,6 \\
\hline V y BD & 54,1 & 56,6 & 60,0 & 48,8 \\
\hline V y AD & 54,5 & 56,9 & 60,4 & 46,7 \\
\hline Error estándar & 0,9 & 1,0 & 1,4 & 0,9 \\
\hline \multicolumn{5}{|c|}{ ANDEVA (Probabilidades) } \\
\hline Color (C ) & 0,1280 & 0,9170 & 0,1542 & 0,6313 \\
\hline densidad (D) & 0,1908 & 0,0964 & 0,6044 & 0,1285 \\
\hline Interacción C x D & 0,6526 & 0,0380 & 0,9699 & 0,6905 \\
\hline \multicolumn{5}{|c|}{ Firmeza del fruto $(\mathrm{N})$} \\
\hline A y BD & 3,9 & 5,6 & 5,2 & 4,6 \\
\hline A y AD & 4,0 & 5,3 & 5,3 & 4,6 \\
\hline R y BD & 4,2 & 5,4 & 5,4 & 4,6 \\
\hline R y AD & 3,9 & 5,3 & 5,4 & 4,6 \\
\hline V y BD & 3,8 & 5,3 & 5,2 & 4,6 \\
\hline V y AD & 3,8 & 5,2 & 5,3 & 4,6 \\
\hline Error estándar & 0,1 & 0,1 & 0,1 & 0,1 \\
\hline \multicolumn{5}{|c|}{ ANDEVA (Probabilidades) } \\
\hline Color (C ) & 0,3035 & 0,1047 & 0,4717 & 0,9139 \\
\hline Densidad (D) & 0,6458 & 0,0919 & 0,6226 & 0,5743 \\
\hline Interacción C x D & 0,7042 & 0,6854 & 0,9994 & 0,9448 \\
\hline \multicolumn{5}{|c|}{ Grados Brix (\% de solidos solubles) } \\
\hline A y BD & 19,8 & 16,5 & 17,7 & 19,3 \\
\hline A y AD & 19,4 & 16,3 & 16,3 & 18,4 \\
\hline R y BD & 19,2 & 15,8 & 17,0 & 18,6 \\
\hline R y AD & 19,1 & 16,5 & 16,5 & 19,4 \\
\hline V y BD & 19,4 & 15,9 & 17,2 & 18,2 \\
\hline V y AD & 18,9 & 16,8 & 15,8 & 18,4 \\
\hline Error estándar & 0,7 & 0,6 & 0,6 & 0,7 \\
\hline \multicolumn{5}{|c|}{ ANDEVA (Probabilidades) } \\
\hline Color (C) & 0,7263 & 0,9051 & 0,8578 & 0,5765 \\
\hline Densidad (O) & 0,5338 & 0,3843 & 0,1010 & 0,8836 \\
\hline Interacción C x D & 0,9514 & 0,6296 & 0,7811 & 0,4490 \\
\hline \multicolumn{5}{|c|}{ Acidez (\% de ácido málico) } \\
\hline A у BD & 0,35 & 0,38 & 0,43 & 0,35 \\
\hline A y AD & 0,36 & 0,36 & 0,42 & 0,34 \\
\hline R y BD & 0,34 & 0,37 & 0,41 & 0,34 \\
\hline R y AD & 0,33 & 0,37 & 0,40 & 0,34 \\
\hline V y BD & 0,31 & 0,36 & 0,42 & 0,33 \\
\hline V y AD & 0,33 & 0,37 & 0,41 & 0,33 \\
\hline Error estándar & 0,01 & 0,01 & 0,01 & 0,01 \\
\hline \multicolumn{5}{|c|}{ ANDEVA (Probabilidades) } \\
\hline Color (C) & 0,0001 & 0,7965 & 0,2883 & 0,1934 \\
\hline Densidad (D) & 0,6458 & 0,6458 & 0,4967 & 0,4319 \\
\hline Interacción C x D & 0,7042 & 0,1715 & 0,8971 & 0,9331 \\
\hline
\end{tabular}

${ }^{1 / C o l o r ~ d e l ~ p o l i e t i l e n o: ~} \mathrm{~A}=\mathrm{azul}, \mathrm{R}=$ rojo, $\mathrm{V}=$ verde. ${ }^{2 /} \mathrm{BD}=$ baja densidad, $\mathrm{AD}=$ alta densidad 


\section{DISCUSIÓN}

Las mediciones de RFA (incidente y transmitida) en los experimentos 1 y 3 se realizaron bajo condiciones climáticas de alta radiación y luminosidad. La RFA transmitida estuvo comprendida para los 3 colores entre 284 a $238 \mu \mathrm{mol}$ $\mathrm{s}^{-1} \mathrm{~m}^{-2}$, menor a aquella indicada por Daza y Cayón (2006) de entre 1631 a $267 \mu \mathrm{mol} \mathrm{s}^{-1} \mathrm{~m}^{-2}$. La RFA incidente medida sobre el dosel de la planta presentó en promedio $2416 \mu \mathrm{mol} \mathrm{s}^{-1} \mathrm{~m}^{-2}$, magnitud menor a aquella indicada por Daza y Cayón (2006) de $2644 \mu \mathrm{mol} \mathrm{s}^{-1} \mathrm{~m}^{-2}$ en las condiciones de Urabá (Colombia) para los mismos colores de fundas usadas en este trabajo. Esta diferencia podría estar dada en función de una mayor cantidad de plantas.ha ${ }^{-1}$ (1750) y una menor RFA transmitida en la zona Atlántica (Costa Rica) con respecto a la menor cantidad de plantas.ha ${ }^{-1}$ (1666) y una mayor RFA transmitida que Daza y Cayón (2006) determinaron al realizar la medición.

La RFA transmitida dentro del racimo presentó un comportamiento similar a los resultados descritos por Daza y Cayón (2006) donde el color azul mostró mayores valores, seguido del rojo y verde. Según los anteriores autores, la magnitud de la longitud de onda que ingresa al interior de la funda está relacionada con el color del polietileno y mencionan que aquellas de color azul tienden a elevar la temperatura en el interior de la funda, las de color rojo dejan pasar una pequeña cantidad del espectro visible sin variar las condiciones internas y las de color verde reducen las longitudes de onda responsables en el aumento de la temperatura en el interior.

No obstante, aún con esta diversidad en la magnitud de la longitud de onda de la RFA transmitida entre los diferentes colores y densidades de polietileno evaluados, no fue posible en esta investigación (experimentos 1 y 3), determinar una respuesta del racimo y sus frutos a dicha variable, contrario con los resultados de (Daza y Cayón 2006, Daza y Cayón 2004, Soto 1992) en bananos (Musa AAA) del subgrupo Cavendish. Dado que en el presente estudio la medición de la RFA transmitida se efectuó inmediatamente después del embolse, sería importante repetir dicha medición inmediatamente antes de la cosecha con el propósito de determinar si en la funda, durante el desarrollo del racimo, merced a su exposición a las condiciones ambientales, ocurre alguna degradación del pigmento de color respectivo que pueda llegar uniformizar la cantidad de RFA transmitida entre los colores de polietileno, como los usados en este experimento.

Los resultados son consistentes en señalar tanto que el peso del racimo como el intervalo de días del embolse a la cosecha así como las dimensiones de los frutos, el color y la firmeza de la cáscara y del fruto, los grados brix y la acidez no fueron afectados por el color ni por la densidad del polietileno de las fundas. En unos pocos casos, algunas de las variables antes mencionadas presentaron diferencias entre tratamientos, situación que dentro el contexto general del estudio no representan un aspecto a considerar.

Esta falta de respuesta productiva y de calidad poscosecha es congruente con lo indicado por Weerasinghe y Ruwanpathirana (2002) quienes indican que el color y tipo del material de embolse no afectaron el intervalo de días de embolse a cosecha, el peso del racimo o de sus frutos ni los grados brix.

Al respecto, es importante mencionar, que el desarrollo de la metodología de campo en los diferentes experimentos que conforman este trabajo, fue similar en todos los casos. De esa manera, en cada experimento los tratamientos se aplicaron en grupos de racimos con una misma edad de embolse (banano y plátano), con una misma cantidad de manos a la floración o al embolse (banano), asignándose dichos tratamientos de manera rotativa y aleatoria conforme la aparición del tamaño de racimo requerido y con la mayor cantidad posible de repeticiones, cada una de las cuales estuvo constituida por un racimo.

Esto permitió eliminar las fuentes de invalidación dadas por el clima, el suelo y el vigor de la planta así como fortalecer el análisis de los datos en función de una comparación entre manos y racimos equivalentes (Vargas y Blanco 2000). Además, la cosecha se efectuó conforme el 
grupo de racimos de la misma edad alcanzaron, en etapas sucesivas, el grosor requerido de cosecha, modalidad que particularmente para bananos es la utilizada para frutos de exportación.

Por el contrario, cuando en este tipo de experimentos cada tratamiento está constituido por un grupo de plantas conformadas en parcela, la expresión de las fuentes de invalidación antes mencionadas puede tener una mayor influencia sobre los resultados. Además, bajo esta perspectiva generalmente la cantidad de repeticiones $o$ parcelas y de las plantas que las conforman es menor, situación que afecta la consistencia del análisis de los datos, que ya de por sí, presentan una alta variación. Asimismo, otra fuente de invalidación común consiste en el uso de una modalidad de cosecha diferente a la comercial, especialmente en el caso de banano, sin tomar en cuenta las etapas sucesivas ni el grosor requerido y solamente un mismo tiempo de cosecha para el grupo de racimos de una misma edad.

La apariencia del racimo de banano tampoco fue afectada por el color y la densidad del polietileno usado y, en aquellos casos en que hubo lesiones, estas fueron grado 2 (con pocas manchas o lesiones pequeñas). Sin embargo, esta variable fue afectada en el plátano, especialmente con la funda de baja densidad, cuyas lesiones en ambos casos, incluyeron los grados 5 (con muchas manchas o lesiones grandes) y 6 (totalmente cubierto de manchas o lesiones-tejido necrosado). En adición, la cantidad de hojas con las que se cosecharon las plantas de todos los experimentos en banano (de 4 a 6 hojas.planta $^{-1}$ ), fue mucho mayor que el de las plantas del experimento de plátano (de 1,5 a 2 hojas.planta ${ }^{-1}$ ), situación que incidió en que la protección del racimo embolsado a la radiación solar fuera también mayor en el primer caso. Ello junto con la conformación particular en el caso del plátano de las primeras manos del racimo que facilita la acumulación de agua en la concavidad que forma sobre éstas el polietileno y, al uso de fundas de $\mathrm{u} \mathrm{Al}$ respecto y para banano Weerasinghe y Ruwanpathirana (2002) indican que el color del material influenció la apariencia externa del racimo y los racimos cubiertos con fundas sin color (transparente) fueron propensos a daños por quema de sol de un ancho similar a las utilizadas en banano pudo provocar la expresión de las lesiones observadas en el plátano. Una condición similar de daño por quema de sol fue observada por Weerasinghe y Ruwanpathirana (2002) en un área de banano sembrada en alta densidad de población (3333 plantas.ha ${ }^{-1}$ ) en donde independientemente del color del polietileno, las lesiones se observaron en aquellas manos o secciones del raquis que permanecieron en contacto con las depresiones con agua formadas por el polietileno, daño que se agravó en las fundas sin color (transparentes). Los anteriores autores indican que en fundas con color la penetración de la luz solar no es tan intensa, y por ende, también el daño por quema de sol.

Al respecto Daza y Cayón (2004) indican que en experimentos con bolsas de diferente color hubo una quema generalizada del raquis de los racimos de plátano por el sol, debido al menor dosel de la planta de plátano que favorece una exposición directa a la luz ultravioleta, que en combinación con el agua que se deposita o condensa sobre la bolsa provoca la quemadura del tejido. Ante estas consideraciones es probable que la diferencia de 2,5 $\mu$ de más en el grosor del polietileno de la funda lechosa de color rojo fuera también factor de influencia sobre la mejor apariencia del racimo de plátano con respecto a la bolsa transparente del mismo color. Al respecto, Soto (1992) indica que en banano el diferente grosor del polietileno no afectó el desarrollo del racimo y que su selección estaría más en función del costo del material o de áreas de cultivo con altas temperaturas.

En plátano también existió la diferencia en el patrón de perforación antes mencionado entre las bolsas de color rojo con $2 \mathrm{~mm}$ de menos en la bolsa de alta densidad. Sin embargo este podría no ser un aspecto importante para definir diferencias entre tratamientos ya que independientemente de su color y de su densidad, todas las bolsas muestran un patrón de perforación en toda su superficie. Esto es consecuente con lo señalado por Soto (1992) quién indica que los racimos con 
bolsas sin perforar, con perforaciones solo en la parte inferior o solo en la parte superior mostraron muy alta incidencia de hongos y que por el contrario, las bolsas perforadas en su totalidad fueron las que mostraron los mejores resultados.

Dado que el insecticida impregnado en la funda varió en algunos tratamientos, dicha situación se trató de mitigar mediante la colocación de cintas de polipropileno impregnadas con el insecticida clorpirifos al $1 \%$ al embolse y 6 semanas después en la totalidad de los mismos. Sin embargo, ello pudo constituir un factor de variación en el caso del plátano y de las fundas de color rojo, donde los racimos con bolsa lechosa e impregnada con bifentrina y cinta con clorpirifos presentaron una mejor apariencia que aquellos con bolsa impregnada con clorpirifos y cinta con el mismo insecticida. Esto es respaldado por la mejor apariencia del racimo de las bolsas azules (bifentrina y clorpirifos) con respecto a las verdes (clorpirifos). La colocación de cintas de polipropileno se efectuó solamente con su impregnación con clorpirifos, toda vez que al contario de la bifentrina, éste es capaz de liberarse dentro de la bolsa.

Ante tal perspectiva, podría ser posible la identificación de racimos de edad similar, mediante el embolse de los mismos con una secuencia semanal de colores de fundas, tal como se informa como una estrategia para el control de la edad del racimo usada por productores en Australia (Daniells y Lindsay 2005). Esto podría brindar una mejor apreciación y localización de los racimos por parte del cortador que las cintas de polipropileno para encinte tradicionalmente usadas con el consiguiente aumento en el rendimiento y en la eficiencia de la labor. Para ello, sería necesaria la evaluación de nuevos colores de polietileno, los cuales junto con aquellos ya evaluados (azul, rojo y verde) puedan conformar la secuencia semanal necesaria.

En la actualidad, la funda de color azul es universalmente usada en la actividad bananera, color elegido, de acuerdo con Wade et al. (1993) para limitar al máximo la radiación ultravioleta que causa quemaduras en la cáscara del fruto. No obstante, fundas de polietileno con diferentes características son evaluadas constantemente con el propósito de mejorar el desarrollo, la presentación y la calidad poscosecha del racimo y sus frutos. Una de ellas, de polietileno transparente y con un grosor de casi el doble $(20,3 \mu)$ de la funda azul convencional $(12,7 \mu)$, contiene un aditivo que le permite filtrar parte de la luz ultravioleta del sol, así como reducir, el paso al racimo de la luz infrarroja, responsables de las quemaduras en la cáscara del fruto. También el uso de fundas de tela no tejida de polipropileno sin insecticida podría brindar una posible opción productiva y de manejo sostenido y amigable con el ambiente, eso sí, en virtud de su costo (casi el doble de las fundas de polietileno normalmente usadas) la necesidad de su reutilización (Cubillo 2004a).

Adicionalmente es importante incluir en futuras investigaciones aspectos relacionados con la aireación dentro del racimo embolsado mediante la definición de un patrón de perforación en toda la funda que desfavorezca la incidencia del Speckling por hongos así como continuar con la búsqueda y evaluación de sustancias de origen natural (Cubillo 2004b) con las cuales impregnar el polietileno de las fundas.

Los resultados obtenidos son claros en demostrar que, bajo las condiciones agroclimáticas propias del caribe costarricense y de la metodología empleada en el desarrollo de los experimentos, no hubo un efecto del color ni de la densidad del polietileno sobre la productividad de racimos y su apariencia así como las dimensiones, presentación y calidad poscosecha de los frutos de banano y plátano. En el caso del plátano, la apariencia del racimo debe de ser mejor estudiada en función de la densidad del polietileno y de los insecticidas impregnados.

\section{AGRADECIMIENTOS}

Los autores agradecen a la gerencia y al personal de las fincas El Esfuerzo, Verde Azul, La Teresa y Platanera Don Rigo así como al Grupo Olefinas, Grupo Banacol, a la empresa FADASA y a la Sección Agropecuaria de JAPDEVA por la colaboración prestada. 


\section{LITERATURA CITADA}

CAMPBELL S.J., WILLIAMS W.T. 1976. Factors associated with maturity bronzing of banana fruit. Australian Journal of Experimental Agriculture and Animal Husbandry. 16, 428-432.

CAYON G. 2007. Influencia de las bolsas de polietileno sobre el desarrollo y carbohidratos de los frutos de plátano, pp. 13. In: R. Murillo, A.L. Bejarano, H. Morales y J. Pubiano (eds.). Memorias Congreso Mundial de Banano y Plátano. Montenegro, Colombia.

CHILLET M., JANNOYER M. 1996. Selección de bolsas para optimizar las condiciones de crecimiento del banano. InfoMusa 5(1):25-27.

CUBILLO D. 2004a. Evaluación semicomercial de la funda Agriban ${ }^{\circledR}$ para la protección del racimo de banano, pp. 60-64. In: A. Vargas y R. Vargas (eds.). Informe Anual 2003. Dirección de Investigaciones. Corporación Bananera Nacional (CORBANA, S.A.). San José, CR.

CUBILLO D. 2004b. Evaluación de fundas de polietileno impregnadas con diferentes sustancias: clorpirifos+azufre, bifentrina y chile+ajo y una funda de polipropileno semicomercial (Agriban ${ }^{\circledR}$ ) usadas para la protección del racimo de banano, pp. 64-66. In: A. Vargas y R. Vargas (eds.). Informe Anual 2003. Dirección de Investigaciones. Corporación Bananera Nacional (CORBANA, S.A.). San José, CR.

DADZIE B.K., ORCHARD J.E. 1997. Evaluación rutinaria postcosecha de híbridos de bananas y plátanos: criterios y métodos. Guías técnicas de INIBAP. IPGRI, INIBAP. 63 p.

DANIELLS J.W., LINDSAY S. 2005. Banana bunch covering. Consultado 21 enero 2010. Disponible http://www2.dpi.qld.gov.au/horticulture/4988. html. DPI note. Department of Primary Industries. Queensland, Australia.

DANIELLS J.W., LISLE T., O'FARREL P.J. 1992. Effect of bunch covering methods on maturity bronzing, yield and fruit quality of bananas in north Queensland. Australian Journal of Experimental Agriculture. 32, 121-125.

DANIELLS J.W., O'FARREL P.J., MULDER J.C., CAMPBELL S.J. 1987. Effect of bunch covering and bunch trimming on bananas in north Queensland. Queensland Journal of Agricultural and Animal Sciences. 44(2):101-105.
DAZA M.A., CAYON G. 2004. Influencia del color de las bolsas de polietileno sobre las características físicas y químicas de los frutos de plátano y banano en época de verano e invierno en tres localidades (norte-centro-sur) de la región de Urabá. Universidad Nacional de Colombia. Sede Bogotá. Facultad de Agronomía. Informe final. $72 \mathrm{p}$.

DAZA M.A., CAYON G. 2006. Efecto del color de las bolsas de polietileno sobre las características físicas y químicas de frutos de banano, pp. 319. In: E. Soprano, F. Adami, L. Lichtemberg, M. Silva (eds.). XVII Reunião Internacional da Associação nas Pesquisas sobre banana no Caribe e na América Tropical. ACORBAT, Santa Catalina, Brasil.

GALAN SAUCO V., CABRERA J., GOMEZ P.M. 1996. The evaluation of different bunch covers (Musa acuminata) in the Canary Islands. Fruits 51(1):13-24.

HEENAN D.P. 1973. Bunch covers for bananas in the Northern District Papua New Guinea. Agricultural Journal. 24(4):156-161.

JANNOYER M., CHILlET M. 1998. Amélioration de la croissance des bananes par utilization de la gaine Katryx ${ }^{\circledR}$.

JOHNS G.G. 1996. Effects of bunch trimming and double bunch covering on yield bananas during Winter in New South Wales. Australian Journal of Experimental Agriculture. 36, 229-235.

LARA F. 1970. Problemas y procedimientos bananeros en la zona Atlántica de Costa Rica. Imprenta Trejos. San José, Costa Rica. 278 p.

ROBINSON J.C. 1996. Bananas and Plantains. CAB International, Wallingford, WK. 238 p.

SERRANO E., SEGURA R., ORTEGA R., SANDOVAL J. 2008. Modelo de restitución del potasio removido en la fruta fresca exportada en una plantación de banano de alta productividad, pp. 106-110. In: J. Sandoval (eds.) Informe Anual 1997. Dirección de Investigaciones. Corporación Bananera Nacional (CORBANA, S.A.). San José, CR.

SOTO M. 1992. Siembra y operaciones de cultivo, pp. 211-365. In: M. Soto (ed.) Bananos: cultivos y comercialización. $2^{\text {a }}$ ed. Litografía e Imprenta LIL. San José, Costa Rica.

VARGAS A., BLANCO F. 2000. Consideraciones metodológicas para la evaluación del desmane en banano (Musa AAA, cv. 'Valery). InfoMusa. 9(2):1921. 
VARGAS A., BLANCO F. 2004. Metodologías para estimar la intensidad de desmane en racimos de banano (Musa AAA, cv. Valery). CORBANA 30(57):107119.

VON LOESECKE H. 1950. Bananas. $2^{\text {da }}$ edición. InterScience, New York.
WWEERASINGHE S.S., RUWANPATHIRANA K.W. 2002. Influence of bagging material on bunch development Banana (Musa spp.) under high density planting system. Annals of Sri Lanka Department of Agriculture. 4:47-53 